\title{
¿CÓMO MEDIR LA CAPACIDAD DINÁMICA DE INNOVACIÓN EN LAS ORGANIZACIONES?*
}

\author{
How to Measure the Dynamic Capability of Innovation in \\ Organizations?
}

\section{RESUMEN}

El conocimiento ha sido una de las herramientas con las que ha contado el ser humano para observar, aprender y evolucionar en cuanto a los retos manifestados en las adaptaciones a las diferentes formas de organización social. Por esto, las Universidades en Colombia no son ajenas a este escenario, aun cuando a través de sus Grupos de Investigación hacen parte de los actores nacionales de innovación. Y justamente por esta razón, se identifica la necesidad de presentar un artículo a través del cual se evidencien los resultados de una revisión bibliográfica de carácter descriptivo en torno a las capacidades dinámicas y equipos de trabajo desde el enfoque organizacional, con el objeto de aportar a la definición de un indicador útil que mida y oriente la capacidad de innovación en los grupos de investigación colombianos, en el camino de configurarlos como micro-ecosistemas de innovación que se anticipen a las realidades del entorno con productos y procesos de valor $y$ uso competitivo.

Palabras clave: Capacidades dinámicas, innovación, gestión del conocimiento, grupos de investigación.

\begin{abstract}
Knowledge has been a tool that allowed humans beings to observe their environment, to learn and evolve in order to face the challenges of their lives and also to adapt to the different and endlessly changing forms of social organizations. For the case of Colombian Universities, which are not different to other types of educational organizations and specifically for research groups, which are key players for innovation at national level, it is essential to present a review from the organizational point of view, taking into account their dynamical and teamwork capabilities, so as to define a useful indicator which measures and guides the capability of innovation of Colombian research groups with the intention of organize them
\end{abstract}

\footnotetext{
Este capítulo es resultado del proyecto de tesis doctoral "Capacidad Dinámica de Innovación y Prácticas de Gestión del Conocimiento en los Grupos de Investigación de las Universidades Colombianas" desarrollada en el marco del Doctorado de Gestión de la Universidad EAN.
} 
GESTIÓN DEL CONOCIMIENTO Y CAPACIDAD DE INNOVACIÓN

Modelos, Sistemas y Aplicaciones

as innovative micro-systems capable to anticipate to contextual reality providing processes and products with added and competitive value for their organizations.

Keywords: Dynamic capabilities, innovation, knowledge management, research group.

\section{INTRODUCCIÓN}

La sociedad del conocimiento ha convertido paulatinamente al conocimiento organizacional en una fuente de ventajas competitivas, ya que es a través de este que se generan propuestas de valor que permiten acometer los desafíos actuales del entorno y el mercado. La habilidad de transformar el conocimiento organizacional en bienes, servicios y procesos innovadores, es lo que en este documento denominamos capacidad dinámica de innovación. Dicha capacidad permite medir en términos de resultados la destreza de una organización para implementar prácticas de gestión de conocimiento y metodologías de aprendizaje, como etapas del proceso de creación de propuestas de valor.

En virtud de lo anterior, este capítulo de libro busca proponer un constructo conceptual que permita evaluar la capacidad dinámica de innovación en las organizaciones a través de la definición conceptual de la capacidad dinámica de innovación, la presentación de los tres retos que tiene una organización para generar esta capacidad, y finalmente, la propuesta de un esquema conceptual que permitirá medir los componentes y variables de la capacidad en las organizaciones.

\section{CAPACIDAD DINÁMICA DE INNOVACIÓN}

Autores como Selznick (1948), Wernerfelt (1984), Schoemaker (1992), Zahra, Sapienza \& Davidsson (2006), Acosta, Longo-Somoza \& Fischer (2013), y Tzortzaki \& Mihiotis (2014), han buscado que las organizaciones comprendan la importancia de los recursos y las capacidades como fuentes de ventaja competitiva, en la medida que se transformen en bienes y servicios que eleven la anticipación y novedad en el mercado o entorno. Por estas razones, y especialmente por el planteamiento realizado por 
Wernerfelt (1984) con la teoría de los recursos y capacidades, emergen las capacidades dinámicas como estrategia para potenciar, adaptar, modificar, y alterar los atributos de una organización, para generar propuestas de valor.

Es asi, que Teece, Pisano \& Shuen (1997) denominaron las capacidades dinámicas como la habilidad de una empresa para integrar, construir y reconfigurar sus competencias internas y externas para adecuarse a entornos de rápido cambio, a través de nuevas e innovadoras formas de ventaja competitiva. Las capacidades dinámicas no son fáciles de imitar (Teece, 2007; 2009) ya que al hacer parte de la estrategia en una organización (Roncancio, 2011), construyen escenarios de colaboración y generan nuevos saberes organizacionales (Garzón, 2015) a través de procesos de exploración y explotación para transformar los recursos y habilidades (Acosta \& Fischer, 2013) en propuestas competitivas (Sánchez, 2016a).

En este sentido, las capacidades dinámicas son una destreza organizacional que facilita la adaptación de las organizaciones al entorno, debido a que motivan interacciones funcionales que forman, conforman, construyen, reconfiguran y movilizan los recursos intelectuales, estructurales y relacionales para responder anticipada y competitivamente al entorno a través de bienes, servicios o procesos innovadores (Sánchez, 2016a).

Existen actualmente muchos abordajes para las capacidades dinámicas en el entorno organizacional, que buscan mejorar su entendimiento a través de la forma que pueden tomar. González, López, Sáez y Verde (2009) plantean tres corrientes: la capacidad dinámica como contingencia para incrementar la efectividad de las respuestas al entorno; la capacidad dinámica como mayoritaria en la alteración y recombinación de recursos, y la capacidad dinámica de innovación como facultad de producir bienes y servicios.

Teniendo en cuenta lo expuesto por González, et al. (2009), planteamos que la mejor manera para demostrar que las organizaciones están haciendo un uso eficiente y efectivo de sus recursos, es evaluar su habilidad 
para generar productos, servicios, y procesos que evidencien innovación, anticipación, y aceptación en el mercado; lo cual se denomina capacidad dinámica de innovación.

Entender la innovación como capacidad dinámica, no solo debe limitarse a comprenderla como proceso, función, tiempo o sistema social (Garzón, 2015) sino ampliar su concepto como capacidad y madurez de una organización para transformar el conocimiento de la misma y de sus colaboradores en conocimiento explicito, codificado, y materializado a través de rutinas, productos y servicios con alto valor agregado.

El conocimiento del que se habla en el párrafo anterior, es posible interpretarlo como un objeto de la realidad a la espera de ser descubierto, capturado y codificado por el sujeto humano (visión funcionalista); o como una construcción social mediada por experiencias intersubjetivas donde juegan un papel primordial el lenguaje, los significados y el contexto en el que se producen estas interacciones (visión interpretativa) (Pérez, 2012). La visión funcionalista y la interpretativa del conocimiento, nos lleva a analizar el cómo se conoce y genera el conocimiento organizacional, denominado por Nonaka \& Takeuchi (1995) la dimensión epistemológica (conocimiento tácito y conocimiento explicito) y ontológica (individual, grupal, organizacional e interorganizacional) del conocimiento (Sánchez, 2016c).

A partir del planteamiento de innovación como capacidad dinámica, Sánchez (2016a) nos muestra que se han desarrollado amplias investigaciones que han posicionado la innovación bajo esta perspectiva (Burns \& Stalker, 1961; Cohen \& Levinthal (1990); Adler \& Shenbar (1990); Kogut \& Zander (1992); Szeto (2000); Neely, Filippini, Forza, Vinelli, \& Hii (2001); Lawson \& Samson (2001); Zhao, Tong, Wong, \& Zhu, 2005; Acosta \& Fischer, 2013).

En términos generales, la capacidad dinámica de innovación evalúa la habilidad de entender, comprender, y transformar los atributos internos de una organización y las necesidades del entorno, en la forma de propuestas de valor y competitividad. 


\section{RETOS DE LA CAPACIDAD DINÁMICA DE INNOVACIÓN: GESTIÓN DEL CONOCIMIENTO, EQUIPOS DE TRABAJO Y METODOLOGÍAS DE APRENDIZAJE}

Como vemos, el conocimiento es conocido y generado por los individuos que sumados hacen las organizaciones, es decir, conocimiento organizacional. En palabras de Nonaka \& Takeuchi (1995) crear comunidades de interacción, motiva el proceso social de conversión del conocimiento tácito (experiencias personales $\mathrm{u}$ organizacionales, modelos mentales, entre otros) a la generación de conocimiento explicito (conceptos, teorías, políticas, manuales, protocolos, portafolio de bienes y servicios) a través de lo que ellos denominaban espiral de conversión de conocimiento, donde no solo hay transformación del conocimiento sino que interactúan todos los niveles de la organización, y luego dichos niveles con el entorno.

Por ello, entre los retos de una organización para generar capacidad dinámica de innovación, se encuentran la necesidad de garantizar prácticas, escenarios y metodologías de aprendizaje que faciliten la interacción del conocimiento de los individuos y el obtenido por la organización en sus diferentes niveles mediante rutinas y saberes.

Las prácticas deben estar orientadas a gestionar la transformación de ese conocimiento organizacional en bienes y servicios, a través de herramientas e instrumentos que fomenten el aprendizaje organizacional, y activen el saber hacer de las organizaciones (Monagas-Docasal, 2012). Lo anterior es nominalmente definido como la gestión del conocimiento, la cual reivindica el papel del conocimiento como fuente de competitividad en las organizaciones, presentándolo como recurso estratégico, el cual puede ser medido a través de la capacidad de generar bienes y servicios innovadores en una organización.

En este sentido, la concepción abstracta del conocimiento se transforma en una concepción tangible, ya que si entendemos el conocimiento como recurso organizacional, es necesario transformarlo en portafolios de bienes y servicios, que nos permite medir nuestra capacidad dinámica de 
generar innovación en la forma de crecimiento del capital humano, estructural y relacional; requiriendo para ello, prácticas de gestión de conocimiento como mapas y directorios del conocimiento, sistemas de gestión de información y monitores, conformación de equipos de trabajo, responsabilidades compartidas, bancos de ideas, vigilancia tecnológica (Acosta, 2010) y recompensas e incentivos.

Por estas razones y tal como lo manifiestan autores como Rodríguez \& Gairín, 2015; Garzón, 2015; Acosta \& Fischer, 2013; García \& Ferrer, 2012; Pinto-Prieto, Becerra-Ardila \& Gómez-Flórez, 2012 la gestión del conocimiento como la suma de prácticas (herramientas, instrumentos, y técnicas) posibilita en las labores diarias y cotidianas, la generación de conocimiento organizacional como fuente de ventaja competitiva, en la medida que fomenta la interacción y reconfiguración del saber hacer con las necesidades que demanda el mercado o entorno en el que se desempeñe la organización; mejora y acelera la capacidad de respuesta a través de la anticipación, y agrega valor desde la innovación a los bienes y servicios (Sánchez, 2016c)

Los escenarios compartidos, son el segundo reto para la generación de la capacidad dinámica de innovación en las organizaciones. La conformación de equipos de trabajo es una práctica de la gestión del conocimiento que, debido a su importancia, se configura como una condición facilitadora en la aplicación e implementación de herramientas e instrumentos que motivan el uso, aplicación, y transferencia del conocimiento para favorecer la innovación organizacional (García \& Cordero, 2010).

Senge (1990) manifiesta que la orientación al cambio es más efectiva por etapas y niveles, aplicando prácticas o herramientas de gestión de conocimiento en grupos de personas o equipos de trabajo, que estén en condiciones de generar resultados alineados con la misión y visión organizacional.

No obstante, es importante resaltar que generar comunidades de interacción solamente se puede hacer realidad a través de equipos de trabajo y no en grupos, ya que en los últimos, cada integrante trabaja para cumplir 
objetivos o actividades específicas (Uribe, Molina, Contreras \& Barbosa, 2013). El propósito es conformar equipos donde dos o más individuos se reúnen para realizar tareas relevantes desde la perspectiva organizacional que surgen de metas y propósitos compartidos con límites y vínculos al amplio contexto precedente del entorno (Kozlowski \& Illen, 2006).

Olmsted (1991) manifestó que las acciones de los equipos de trabajo se caracterizan por basarse en compromiso colectivo, interacción social, creación de una conciencia recíproca y estabilidad de las relaciones en el tiempo (Sánchez, 2016b). La utilidad de los equipos de trabajo como práctica de gestión de conocimiento, radica en la obtención de altos estándares de calidad dentro de la gestión organizacional, y la eficacia en la generación de situaciones que facilitan la generación de capacidades dinámicas, especialmente la de innovación (Aleida \& Heriberto, 2003).

En relación a las metodologías de aprendizaje, se presentan dos perspectivas que apoyarían la implementación de prácticas de gestión de conocimiento en el marco de equipos de trabajo. La primera denominada STI (Ciencia y Tecnología basada en la Innovación) orientada a generar resultados en términos de innovación y el desarrollo, y la segunda, DUI (Haciendo, Usando e Interactuando) más enfocada al proceso de creación de nuevo conocimiento.

La metodología STI se refiere específicamente a la forma en que las organizaciones utilizan y desarrollan la ciencia como conocimiento en el contexto de sus actividades innovadoras, relacionada más con una concepción lineal del proceso de innovación en las organizaciones (Parrilli, 2010; Jensen, Johnson, Lorenz, \& Lundvall, 2007).

Por su parte, la metodología DUI se centra en el conocimiento tácito, más exactamente en el know-how (conocimiento construido a partir de la experiencia y habilidades prácticas) y know-who (conocimiento generado a partir de las relaciones interpersonales) (Foray \& Lundvall, 1998). El DUI, como metodología fue diseñada para observar y medir los grados de colaboración de los acuerdos suscritos entre empresa, clientes, proveedores y 
sectores de la competencia, soportando estos procesos en una estructura de aprendizaje que posibilite la transformación de activos del conocimiento analítico y científico en nuevo 'conocimiento sistémico' (González-Pernía, Parrilli y Peña-Legazkue, 2015; Jensen, Johnson, Lorenz, \& Lundvall, 2007).

La metodología DUI promueve y prioriza el aprendizaje organizacional a través de prácticas rutinarias y políticas específicas, como estrategia de generación de conocimiento e innovación al interior de las organizaciones. Dicho aprendizaje se fortalece desde la existencia de estructuras organizacionales de carácter horizontal y flexible, y la estimulación al intercambio de conocimientos, lo cual debe propender en las mejoras de los bienes y servicios ofrecidos por las empresas en el mercado (Parrilli, 2010).

La combinación de las dos metodologías refuerzan las condiciones ideales para generar capacidad dinámica de innovación en las organizaciones; DUI permite identificar actores y saberes del conocimiento organizacional a través de las prácticas de gestión de conocimiento, y STI orienta a los equipos de trabajo a generar resultados con componentes de ciencia, tecnología e innovación. Así de esta manera, las prácticas de gestión de conocimiento, los equipos de trabajo, y las metodologías de aprendizaje, se convierten en los retos de una organización, si quiere generar capacidad dinámica de innovación.

\section{4. ¿CÓMO MEDIR LA CAPACIDAD DINÁMICA DE INNOVACIÓN?}

En la academia y la empresa se encuentran diversos modelos, métricas, e indicadores que evidencian tanto el grado de innovación de un bien o servicio como la innovación como capacidad dinámica en las organizaciones; algunos de ellos son:

1. El Manual de Bogotá como instrumento de Normalización de Indicadores de Innovación Tecnológica en América Latina y el Caribe (Jaramillo, Lugones y Salazar, 2001);

2. Eficiencia de la innovación (Hamel \& Getz, 2004); 
3. Observación de componentes y dimensiones del proceso de innovación (Molina-Castillo y Munuera-Alemán, 2008);

4. Clima organizacional para la innovación (Anderson, 2008);

5. Modelo integral de innovación en las organizaciones (Lawson \& Samson, 2001);

6. Recursos Internos y Externos para la Capacidad de Innovación (Romijn \& Albaladejo, 2002);

7. Factores Organizacionales (Koc, 2007); y

8. Recursos y Desempeño Innovador (Urgal, Quintás, \& Arévalo, 2011).

Sin embargo, no se encontró en la revisión de literatura una métrica o sistema de indicadores que evaluara la capacidad dinámica de innovación en las organizaciones. En virtud de lo anterior, a partir de los hallazgos identificados en este documento, proponemos el siguiente constructo conceptual, compuesto por dos componentes: prácticas de gestión del conocimiento-PGC, y metodologías de aprendizaje-MA, y once variables que podrían medir la capacidad dinámica de innovación en las organizaciones:

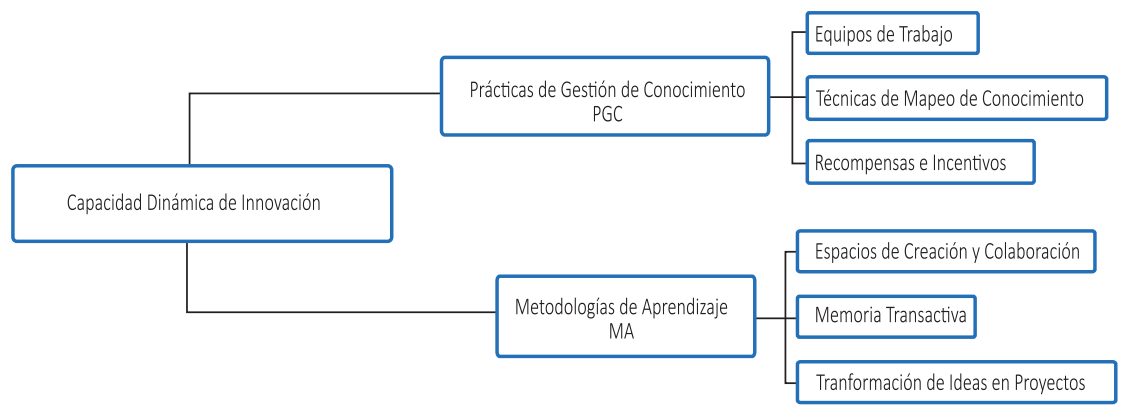

Gráfico 1. Constructo: Capacidades Dinámicas de Innovación

Fuente: Elaboración propia

A continuación, se detallan las variables y autores que han soportado este planteamiento. 
GESTIÓN DEL CONOCIMIENTO Y CAPACIDAD DE INNOVACIÓN

Modelos, Sistemas y Aplicaciones

Tabla 1. Componentes y Variables del Constructo: Capacidades Dinámicas de Innovación

\begin{tabular}{|c|c|c|}
\hline COMPONENTE & VARIABLE & AUTORES \\
\hline $\begin{array}{l}\text { Prácticas de } \\
\text { Gestión del } \\
\text { Conocimiento }\end{array}$ & $\begin{array}{l}\text { Existencia de Equipos de Trabajo } \\
\text { Auto-asignación de propósitos y metas } \\
\text { específicas de equipo: concepción, vi- } \\
\text { sión y uso del conocimiento del equipo } \\
\text { y su sinergia con la organización } \\
\text { Heterogeneidad como principio en la } \\
\text { visión de equipo } \\
\text { Mapeo y Portafolio del Conocimiento } \\
\text { Protocolo de asunción de riesgos desde } \\
\text { el enfoque ensayo-error } \\
\text { Instrumentos de monitoreo de entorno } \\
\text { Sistema de recompensas e incentivos a } \\
\text { la innovación }\end{array}$ & $\begin{array}{l}\text { Moreno-Luzón et al. (2001) } \\
\text { Tiwana (2002) } \\
\text { Romin \& Albaladejo (2002) } \\
\text { Chiva-Gómez, Camisón, Zornoza } \\
\text { \& Lapiedra-Alcami (2003) } \\
\text { Gómez \& Acosta (2003) } \\
\text { Roncancio (2011) }\end{array}$ \\
\hline $\begin{array}{l}\text { Metodologías de } \\
\text { Aprendizaje }\end{array}$ & $\begin{array}{l}\text { Espacios dinámicos de aprendizaje y } \\
\text { colaboración organizacional (adqui- } \\
\text { sición, generación y combinación del } \\
\text { conocimiento organizacional) } \\
\text { Memoria Transactiva -sistema de } \\
\text { conocimiento compartido- } \\
\text { Metodologías de identificación y con- } \\
\text { versión de ideas en proyectos }\end{array}$ & $\begin{array}{l}\text { Moreno-Luzón et al. (2001) } \\
\text { Domínguez \& Martins (2014) } \\
\text { Jaramillo, Lugones \& Salazar } \\
\text { (2001) } \\
\text { Guan \& Ma (2003) }\end{array}$ \\
\hline
\end{tabular}

Fuente: Elaboración propia

\section{CONCLUSIONES}

Como resultado de los planteamientos aquí señalados, se puede inferir que el conocimiento no solo genera valor económico; es un valor estratégico y diferenciador en las organizaciones inmersas en este presente competitivo y globalizado, caracterizado por una sociedad que demanda el incremento de la capacidad de respuesta colectiva, y alternativas asequibles para el mejoramiento de la calidad de vida.

Por esta razón, las organizaciones demandan prácticas y metodologías que hagan interactuar el conocimiento de su organización con la informa- 
ción que brinda la dinámica del entorno y del mercado, con el objeto de mejorar la capacidad de respuesta y adaptación a través de la toma de decisiones estratégicas que los lleven a producir bienes y servicios novedosos, útiles, pertinentes e innovadores. Implementar prácticas de gestión de conocimiento y metodologías de aprendizaje hacen que la organización inicie un proceso de cambio, entendiéndose desde la perspectiva de ecosistema de conocimiento, a través de la cual se hace mejor aprovechamiento y uso de sus recursos, se transforman en conocimiento, y se generan propuestas de valor con anticipación y competitividad. Dicho esto, una de las principales herramientas para configurarse como organizaciones de valor, es generar y desarrollar capacidades dinámicas organizacionales, bajo el marco de prácticas de gestión del conocimiento en unidades o equipos de trabajo, donde se potencialice el conocimiento organizacional para dar respuesta a las demandas del entorno desde procesos, bienes o servicios que evidencien una significativa creación de valor (Sánchez, 2017).

En este sentido, evaluar las organizaciones desde el enfoque de la capacidad dinámica de innovación, facilita el establecimiento de lineamientos que mejoren el desempeño a través de la generación de estrategias que faciliten no solo su inserción al mercado sino su posicionamiento, a través de actividades de innovación, investigación y desarrollo experimental, reflejadas en una diversificación y agregación de valor al portafolio de bienes y servicios. Por esto, esperamos que una vez culminada la investigación, logremos construir y validar la batería de indicadores de la capacidad dinámica de innovación para las organizaciones.

\section{REFERENCIAS BIBLIOGRÁFICAS}

Acosta, J. (2010). Creación y desarrollo de capacidades tecnológicas: un modelo de análisis basado en el enfoque de conocimiento. (Tesis doctoral). Universidad Autónoma de Madrid.

Acosta, J. \& Fischer, A. (2013). Condiciones de la gestión del conocimiento, capacidad de innovación y resultados empresariales. Un modelo explicativo. Pensamiento y Gestión, 35, 25-63. 
Acosta, J., Longo-Somoza, M. \& Fischer, A. (2013). Capacidades dinámicas y gestión del conocimiento en nuevas empresas de base tecnológica. Cuadernos de Administración, 26(47), 35-62.

Adler, P., \& Shenbar, A. (1990). Adapting your technological base: the organizational challenge. Sloan Management Review, 32(1), 25-37.

Anderson, A. M. (2008). Review: A framework for NPD management: doing the right things, doing them right, and measuring the results. Trends in Food Science \& Technology, 19, 553-561. doi:10.1016/j.tifs.2008.01.015

Arzola, M. \& Mejías, A. (2010). Morfología del aprendizaje y la gestión del conocimiento del sector servicios de consultoría. Ingeniería Industrial, 9(1), 37-51.

Augier, M., \& Teece, D. (2007). Dynamic capabilities and multinational enterprise: Penrosean insights and omissions. Management International Review, 47(2), 175-192.

Bontis, N. (1996). There's a price on your head: Managing intellectual capital strategically. Business Quarterly, 60(4), 40-78.

Burns, T., \& Stalker, G. (1961). The management of innovation. London: Tavistock Publications.

Chiva-Gómez, R., Camisón-Zornoza, C.,Lapiedra-Alcamí, L. (2003). Organizational learning and product design management: towards a theoretical model. The Learning Organization, 3(10), 167-184.

Cohen, W., \& Levinthal, D. (1990). Absorptive Capacity: A New Perspective on Learning and Innovation. Administrative Science Quarterly, 35(1), 128-152.

Domínguez, R., \& Martins, M. (2014). Knowledge Management: an Analysis from the Organizational Development. Journal of Technology Management \& Innovation, 9(1), 131-147.

Eisenhardt, K., \& Martin, J. (2000). Dynamic Capabilities: What Are They? Strategic Management Journal, 21(10-11), 1105-1121.

Foray, D. \& Lundvall, B. (1998). The knowledge-based economy: from the economics of knowledge to the learning economy. The economic impact of knowledge, 115-121. 
García, A., y Ferrer, M. (2012). Gestión del conocimiento en Cuba: diseminación de sus resultados de investigación, de 1997-2010. Ciencias de la Información, 43(3), 23-32.

García, F., \& Cordero, A. (2007). Los equipos de trabajo: una práctica basada en la Gestión del Conocimiento. Visión Gerencia, 7(1), 45-58.

García, F., Cordero, E (2010). Proceso de Gestión del Conocimiento en Carabobo (Venezuela) y Tamaulipas (México). Pensamiento \& Gestión, 28, 132-154.

Garzón, M. (2015). Modelo de capacidades dinámicas. Dimensión Empresarial, 12(3), 111-131.

Gómez, A. \& Acosta, H. (2003). Acerca del trabajo en grupos o equipos. ACIMED, 11(6), 62-89.

González, J., López, J., Sáez, P., y Verde, M. (2009). Concepto e implicaciones de las capacidades dinámicas desde un enfoque de dirección del conocimiento. XV Congreso de la AECA: Asociación Española de Contabilidad y Administración de Empresas. Valladolid. Recuperado de http://www. aeca1.org/pub/on_line/comunicaciones_xvcongresoaeca/general.htm

González-Pernía, J., Parrilli, M., \& Peña-Legazkue, I. (2015). STI-DUI learning modes, firm-university collaboration and innovation. Journal of Technology Transfer, 40(3), 475.

Guan, J., \& Ma, N. (2003). Innovative capability and export performance of Chinese firms. Technovation, 23(9), 737-747.

Guth, W., \& Ginsberg, A. (1990). Guest Editors Introduction: Corporate Entrepreneurship. Strategic Management Journal, 11(5), 5.

Hamel, G., \& Getz, G. (2004). Cómo innovar en una era de austeridad. Harvard Business Review, 82(8), 10-21.

Helfat, C., Finkelstein, S., Mitchell, W., Peteraf, M., Singh, H., Teece, D., \& Winter, S. (2009). Dynamic Capabilities: Understanding Strategic Change in Organizations. Hoboken: Blackwell Publishing Ltd.

Henao-García, E. A., López-González, M., \& Garcés-Marín, R. (2014). Medi- 
ción de capacidades en investigación e innovación en instituciones de educación superior: una mirada desde el enfoque de las capacidades dinámicas. Entramado, 10(1), 252-271.

Jaramillo, H., Lugones, G. \& Salazar, A. M. (2000). Normalización de indicadores de innovación tecnológica en América Latina y el Caribe: Manual de Bogotá. Red Iberoamericana de Indicadores de Ciencia y Tecnología (RICYT)/Organización de Estados Americanos (OEA)/Cyted Programa/ Colciencias/Ocyt. Bogotá: OEA. COLCIENCIAS.

Jensen, M., Johnson, B., Lorenz, E., \& Lundvall, B. (2007). Forms of knowledge and modes of innovation. Research Policy, 36, 680-693. doi:10.1016/j.respol.2007.01.006

Koc, T. (2007). Organizational determinants of innovation capacity in software companies. Computers \& Industrial Engineering, 53(3), 373-385. doi:http://dx.doi.org/10.1016/j.cie.2007.05.003

Kogut, B., \& Zander, U. (1992). Knowledge of the firm, combinative capabilities, and the replication of technology. Organization Science, 3(3), 383-397.

Kozlowski, S. W., \& Ilgen, D.R. (2006). Enhancing the Effectiveness of Work Groups and Teams. Psychological Science in the Public Interest, 7(3), 77124. doi:10.1111/j.1529-1006.2006.00030.x

Lawson, B., \& Samson, D. (2001). Developing Innovation Capability in Organisations: A Dynamic Capabilities Approach. International Journal of Innovation Management, 5(3), 377-400.

Marín-García, J., \& Zárate-Martínez, M. (2008). Propuesta de un modelo integrador entre la gestión del conocimiento y el trabajo en equipo. Intangible Capital, 4(4), 255-280.

Molina-Castillo, F. J., \& Munuera-Alemán, J. L. (2008). Efectos de la novedad y de la calidad del producto en el resultado a corto y a largo plazo en las empresas innovadoras españolas. Universia Bussiness Review (20), 68-83.

Monagas-Docasal, M. (2012). El capital intelectual y la gestión del conocimiento. Ingeniería Industrial, 33(2), 142-150. 
Moreno-Luzón, M. D., Peris, F.J., González, T. (2001). Gestión de la calidad y diseño de las organizaciones: teoría y estudio de casos. Madrid: Pretince Hall.

Neely, A., Filippini, R., Forza, C., Vinelli, A., \& Hii, J. (2001). A framework for analysing business performance, firm innovation and related contextual factors: perceptions of managers and policy makers in two European regions. Integrated Manufacturing Systems, 12(2), 114-124.

Nelson, R. (1991). Why do firms differ, and how does it matter? Strategic Management Journal, 12(2), 61-74.

Nonaka, I., \& Takeuchi, H. (1995). The knowledge creating company: how Japanese companies create the dynamics of innovation. New York: Oxford University Press.

Olmsted, M. (1971). El pequeño grupo. Buenos Aires: Paidós.

Parrilli, M (2010). Innovación y aprendizaje: lecciones para el diseño de políticas. Seminario sobre Innovación y Aprendizaje. Agencia Vasca de la Innovación- INNOBASQUE e Instituto Vasco de Competitividad- ORKESTRA, Bizkaia.

Pérez, J. (2012). Asociaciones entre madurez de gestión del conocimiento y desempeño innovador: organización y personas e interpretación. Revista Lasallista de Investigación, 9(1), 86-95.

Pineda, L. (2013). Prospectiva estratégica en la gestión del conocimiento: una propuesta para los grupos de investigación colombianos. Investigación y Desarrollo, 21(1), 289-311.

Pinto-Prieto, L., Becerra-Ardila, L., \& Gómez-Flórez, L. (2012). Carencias en los sistemas de gestión del conocimiento: una revisión bibliográfica. El profesional de la información, 21,(3), 268-276.

Pisano, G. (1994). Knowledge, Integration, and the Locus of Learning: An Empirical Analysis of Process Development. Strategic Management Journal, 15(1), 85-100.

Primera, C., Torres, M., Alvarado, H., \& Guerrero, J. (2014). Factores críticos de éxito en la gestión de los equipos de investigación científica universitarios. Compendium, 17(32), 79-100. 
Rodríguez, D., \& Gairín, J. (2015). Innovación, aprendizaje organizativo y gestión del conocimiento en las instituciones educativas. Educación, 24(46), 73-90.

Romijn, H., \& Albaladejo, M. (2002). Determinants of innovation capability in small electronics and software firms in southeast England. Research Policy, 31, 1053-1067. doi:10.1016/S0048-7333(01)00176-7

Roncancio, P. (2011). De las capacidades dinámicas como enfoque de la estrategia a la integración de competencias para la construcción de un entorno colaborativo universidad-empresa. Revista Ciencias Estratégicas, 19(26), 295-306.

Sánchez, D. (2016a). Aportes para un constructo de capacidades dinámicas de innovación para los grupos de investigación colombianos (en proceso de publicación). Revista Electrónica Ide@s Concyteg.

Sánchez, D. (2016b). Grupos de Investigación desde el enfoque de capacidades dinámicas y gestión del conocimiento en las universidades colombianas. 51 Consejo Latinoamericano de Escuelas de Administración CLADEA 2016, Medellín, Colombia.

Sánchez, D. (2016c). Ayer y hoy de la gestión del conocimiento desde el enfoque organizacional: aprendizajes de la evolución en los modelos para un framework inicial. Revista Electrónica Ide@S Concyteg, 133, 21-56.

Sánchez, D. (2017). Capacidad dinámica de innovación y prácticas de gestión del conocimiento en los grupos de investigación de las universidades colombianas. (Tesis doctoral en curso). Universidad EAN, Bogotá, Colombia.

Sawhney, M., Wolcott, R., \& Arroniz, I. (2006). The 12 Different Ways for Companies to Innovate. Sloan Management Review, 47(3), 74-81.

Schmitz, S., Rebelo, T., Gracia, F. \& Tomás, A. (2014). La cultura de aprendizaje y los procesos de gestión del conocimiento: ¿hasta qué punto se relacionan de hecho? Revista de Psicología del Trabajo y de las Organizaciones, 30(3), 113-121.

Schoemaker, P. (1992). How to Link Strategic Vision to Core Capabilities. Sloan Management Review, 34(1), 67-81. 
Selznick, P. (1948). Foundations of the Theory of Organization. American Sociological Review, 13(1), 25-35.

Senge, P. (1990). La quinta disciplina: cómo impulsar el aprendizaje en la organización inteligente. Buenos Aires: Granica.

Serna, L. (2013). Prospectiva estratégica en la gestión del conocimiento: Una propuesta para los grupos de investigación colombianos. Investigación y Desarrollo, 21(1), 237-259.

Szeto, E. (2000). Innovation capacity: working towards a mechanism for improving innovation within an inter-organizational network. The TQM Magazine, 12(2), 149-158.

Teece, D. (2007). Explicating dynamic capabilities: the nature and microfoundations of (sustainable) enterprise performance. Strategic Management Journal, 28(13), 1319-1350.

Teece, D. (2009). Dynamic capabilities and strategic management organizing for innovation and growth. Oxford: Oxford University Press.

Teece, D., \& Pisano, G. (1994). The dynamic capabilities of firms: an introduction. Industrial and Corporate Change, 3(3), 537-556.

Teece, D., Pisano, G., \& Shuen, A. (1997). Dynamic Capabilities and Strategic Management. Strategic Management Journal, 18(7), 509-533.

Templeton, G., Lewis, B., \& Snyder, C. (2002). Development of a Measure for the Organizational Learning Construct. Journal of Management Information Systems, 19(2), 175-218.

Tiwana, A. (2002). The Knowledge Management Toolkit: Orchestrating It. Strategy and Knowledge Platforms. New Jersey: Prentice Hall PTR.

Tzortzaki, A. \& Mihiotis, A (2014). A Review of Knowledge Management Theory and Future Directions. Knowledge \& Process Management, 21(1), 29-41.

Urgal, B., Quintás, M. Á., \& Arévalo Tomé, R. (2011). Conocimiento tecnológico, capacidad de innovación y desempeño innovador: el rol moderador del ambiente interno de la empresa. Cuadernos de Economía y Dirección de la Empresa, 14, 53-66. doi:10.1016/j.cede.2011.01.004 
Uribe, A., Molina, J., Contreras, F., \& Barbosa, D. (2013). Liderar equipos de alto desempeño: un gran reto para las organizaciones actuales. Universidad \& Empresa, 25, 53-71.

Vassiliadis, S., Back, A. \& Krogh, G. V. (2000). Competing with intellectual capital: theoretical background. St. Gallen: Institute for Information Management.

Vick, T., Nagano, M., \& Popadiuk, S. (2015). Information culture and its influences in knowledge creation: Evidence from university teams engaged in collaborative innovation projects. International Journal of Information Management, 35(3), 292-298.

Wernerfelt, B. (1984). A resource-based view of the firm. Strategic Management Journal, 5(2), 171-180.

Zahra, S., \& George, G. (2002). Absorptive Capacity: A review, reconceptualization, and extension. Academy of Management Review, 27(2), 185-203.

Zahra, S., Sapienza, H. \& Davidsson, P. (2006). Entrepreneurship and Dynamic Capabilities: A Review, Model and Research Agenda. Journal of Management Studies, 43(4), 917-955.

Zhao, H., Tong, X., Wong, P., \& Zhu, J. (2005). Types of technology sourcing and innovative capability: An exploratory study of Singapore manufacturing firms. The Journal of High Technology Management Research, 16(2), 209-224.

Zollo, M., \& Winter, S. (2002). Deliberate Learning and the Evolution of Dynamic Capabilities. Organization Science, 13(3), 339-351.

Cómo citar este capítulo:

Sánchez Rodríguez, D. (2017). ¿Cómo medir la capacidad dinámica de innovación en las organizaciones? En K. Barrios Hernández, E. Olivero Vega, J. C. Acosta Prado, I. Meñaca Guerrero, A. M. Cazallo Antúnez, H. J. Medina Carrascal, ... O. Hernández Ariza, Gestión del conocimiento y capacidad de innovación. Modelos, Sistemas y Aplicaciones (pp.105-122). Barranquilla: Universidad Simón Bolívar. 